\title{
Cirurgia de revascularização do miocárdio minimamente invasiva com uso de enxerto composto: relato de caso
}

Fábio B. JATENE*, Paulo PÊGO-FERNANDES*, Hector van Dyck ARBULU*, André Luiz Shinji HAYATA*, Roberto KALIL*, László MOLNÁR*, Adib D. JATENE*

Jatene F B, Pêgo-Fernandes P. Arbulu H D, Hayata A L S, Kalil R, Molnár L, Jatene A D - Cirurgia de revascularizaçāo do miocárdio minimamente invasiva com uso de enxerto composto: relato de caso. Rev Bras Cir Cardiovasc 1996; 11 (4): 307-10.

RESUMO: Este trabalho relata nossa experiência com a utilização de enxerto composto em cirurgia de revascularização do miocárdio minimamente invasiva (CRMMI). Uma alternativa para a CRMMI é o uso de enxertos compostos, nos casos em que se pretende revascularizar vários ramos coronarianos, quando a artéria torácica interna se apresenta curta ou ramifica-se precocemente. Foi operada paciente do sexo feminino, 54 anos, portadora de lesão de $90 \%$ em artéria interventricular anterior em seu terço proximal e que foi submetida à CRMMI por minitoracotomia esquerda. A artéria torácica interna esquerda (ATIE) foi amplamente dissecada utilizando-se bisturi longo, auxílio de ótica de videotoracoscopia e clipe para ligadura dos ramos intercostais. Após a secção da ATIE houve ausência de fluxo sangũineo e evidência de hematoma e dissecção da sua luz no terço distal. Este segmento comprometido foi desprezado e a ATIE foi prolongada por interposição de segmento de veia safena autógena, tornando possivel a anastomose com o ramo interventricular anterior. A operaçāo teve duração de 3 horas. A paciente foi extubada após 4 horas de pósoperatório e o dreno pleural esquerdo foi retirado no $3^{\circ}$ dia; a paciente evoluiu bem, não apresentando intercorrências clínicas pós-operatórias, tendo recebido alta hospitalar 5 dias após a operaçāo. A cineangiocoronariografia pós-operatória comprovou anastomose patente. O estudo com ecodoppler realizado no $4^{2}$ mês de pós-operatório mostrou derivação pérvia e fluxo de alta resistência. Em conclusão, o uso de enxerto composto para CRMMI mostrou ser uma alternativa eficaz para ampliar a extensão da ATIE, possibilitando a anastomose, quando a mesma é curta ou apresenta problemas de fluxo ou, ainda, se se pretende tratar mais de uma coronária

DESCRITORES: Revascularização miocárdica, métodos. Artérias torácicas, cirurgia. Vasos coronários, cirurgia. Toracoscopia. Cirurgia minimamente invasiva.

\section{INTRODUÇÃO}

O uso da artéria torácica interna esquerda para a revascularização da artéria interventricular anterior tem sido amplamente aceito desde as primeiras publicações em 1980, onde se mostrou mais patente em comparação com enxertos de veia safena (1).
O uso da toracotomia esquerda foi aprovado para revascularização do miocárdio e, atualmente, alguns autores, inclusive em nosso meio ${ }^{(2-4)}$ propõem o uso da minitoracotomia para o desenvolvimento da anastomose entre artéria torácica interna esquerda e o ramo interventricular anterior.

Uma alternativa para a revascularização do

Trabalho realizado no Instituto do Coração do Hospital das Clinicas da Faculdade de Medicina da Universidade de São Paulo. São Paulo, SP, Brasil. Recebido para publicaçăo em dezembro de 1996.

* Do Instituto do Coração do Hospital das Clínicas da Faculdade de Medicina da Universidade de São Paulo.

Endereço para correspondência: Fábio B. Jatene. Av. Dr. Enéas de Carvalho Aguiar, 44 - Divisão Cirúrgica. CEP: 05403-000, São Paulo, SP, Brasil. 
Jatene F B, Pêgo-Fernandes P, Arbulu HD, Hayata A LS, Kalil R, Molnar L, Jatene A D - Cirurgia de revascularização do miocárdio minimamente invasiva com uso de enxerto composto: relato de caso. Rev Bras Cir Cardiovasc 1996; 11 (4): 307-10.

miocárdio com o emprego desta técnica é a utilização dos enxertos compostos, nos casos em que se pretende revascularizar várias coronárias, ou quando a artéria torácica interna se apresenta curta ou ramificada precocemente.

Relatamos 1 caso de paciente operado no Instituto do Coração do Hospital das Clínicas da Faculdade de Medicina da Universidade de São Paulo, no qual se utilizou enxerto composto para a revascularização do ramo interventricular anterior.

\section{RELATO DO CASO}

Paciente de 54 anos, sexo feminino, cor bran$\mathrm{ca}$, peso de $61 \mathrm{~kg}, 1,66 \mathrm{~m}$ de altura, em uso de inibidores da ECA, antagonistas do cálcio, ácido acetilsalicílico; dislipidêmica, tabagista. Em junho de 96 apresentou dor precordial e sensação de opressão de curta duração sem relação aos esforços. Foram realizados exame ecocardiográfico e teste de esforço, sendo ambos negativos. No dia 14/07/96 apresentou dor precordial prolongada típica, recorrendo ao Pronto Socorro, sendo evidenciado infarto agudo do miocárdio anterior, com pico de enzima creatinofosfoquinase. fração MB 366 u/l. O eletrocardiograma mostrou supradesnivel de ST em parede anterior, sendo indicada trombólise (rtpa), com sucesso, evoluindo posteriormente com dor precordial. O cateterismo mostrou artéria interventricular anterior sub-ocluida $(90 \%)$ em seu terço proximal, ramo diagonal com lesão menos significativa, além de coronária direita e circunflexa com irregularidades. A ventriculografia revelou hipocinesia ânteroapical.

O exame ecocardiográfico mostrou miocardiopatia segmentar do ventrículo esquerdo, hipocinesia ântero-apical e fração de ejeção de $54 \%$.

No dia $5 / 8 / 96$ foi submetida a revascularização do miocárdio por minitoracotomia esquerda. Realizou-se incisão de aproximadamente $8 \mathrm{~cm}$, a nível do quarto espaço intercostal esquerdo. A artéria torácica esquerda foi amplamente dissecada utilizando-se bisturi longo, auxílio de ótica de videotoracoscopia e clipe para ligadura dos ramos colaterais. Durante a dissecção apresentou quadro de fibrilação ventricular, que foi revertida com cardioversăo elétrica e infusão de xilocaína endovenosa.

Após a secção da artéria torácica interna esquerda em seu terço distal, houve ausência de fluxo sangüineo e evidência de hematoma e dissecção da luz no terço distal. Com a secção da área comprometida a artéria ficou curta, necessitando de emenda de aproximadamente $3 \mathrm{~cm}$ de veia safena, para ser utilizada em anastomose término-terminal com a veia safena e, posteriormente, anastomose na artéria interventricular anterior ao nivel do terço médio (Figura 1). Ambas as suturas foram realizadas com Polipropileno 7-0.

O tempo aproximado de isquemia foi de 12 minutos, utilizando-se fio de Polipropileno 5-0 e torniquete de Polietileno para ocluir-se a artéria interventricular anterior, proximal e distalmente, no momento da anastomose. O tempo total da operação foi de 3 horas, seindo a paciente extubada após 4 horas e 30 minutos no pós-operatório imediato.

Durante as primeiras 24 horas, apresentou, pelo dreno pleural esquerdo, débito de $380 \mathrm{ml}$ de líqüido sero-hemático; o dreno foi retirado no $3^{\circ}$ dia de pósoperatório. Os exames laboratoriais não mostraram alterações, creatinofosfoquinase, fração MB 5 u/l. Não houve intercorrências clínicas pós-operatórias. A cineangiocoronariografia pós-operatória mostrou a anastomose de artéria torácica interna esquerda ao ramo interventricular anterior patente (Figura 2). A paciente recebeu alta hospitalar no $5^{\circ}$ dia de pósoperatório. O estudo com ecodoppler realizado no $4^{2}$ mês de pós-operatório mostrou derivação patente e a análise espectral da curva da velocidade indica leito distal de alta resistência (Figura 3).

\section{COMENTÁRIOS}

A cirurgia de revascularização do miocárdio evoluiu significativamente nos últimos anos e muito se tem discutido a respeito de melhores condições para preservar e recuperar o músculo cardíaco $(5,6)$.

O uso de trombolíticos por via intravenosa ou intracoronária possibilitou melhor evolução dos pacientes com infarto agudo do miocárdio (IAM). Como alternativa de revascularização do miocárdio, a

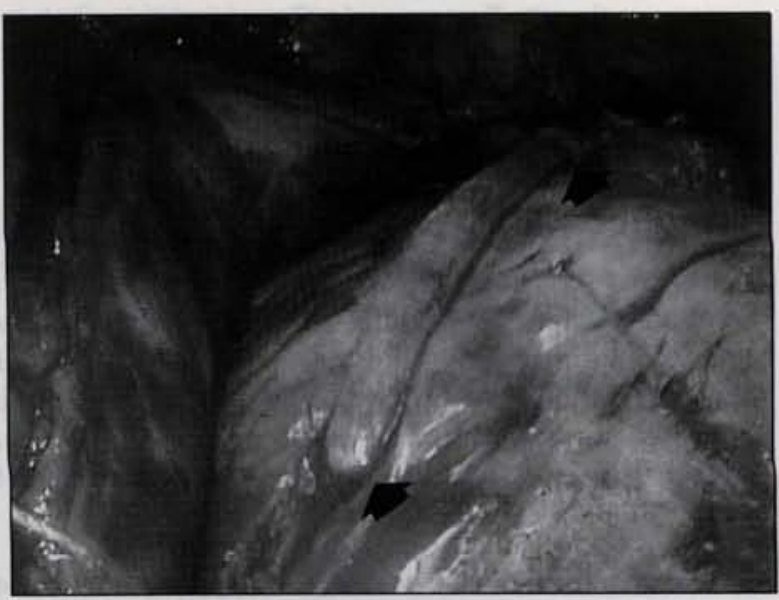

Fig. 1 - Detalhe do campo operatório, observando-se segmento de safena anastomosado à artéria interventricular anterior $e$ anastomose entre a safena e a artéria torácica interna (setas). 
Jatene F B, Pêgo-Fernandes P, Arbulu H D, Hayata A L S, Kalil R, Molnar L, Jatene A D - Cirurgia de revascularização do miocárdio minimamente invasiva com uso de enxerto composto: relato de caso. Rev Bras Cir Cardiovasc 1996;11 (4): $307-10$.

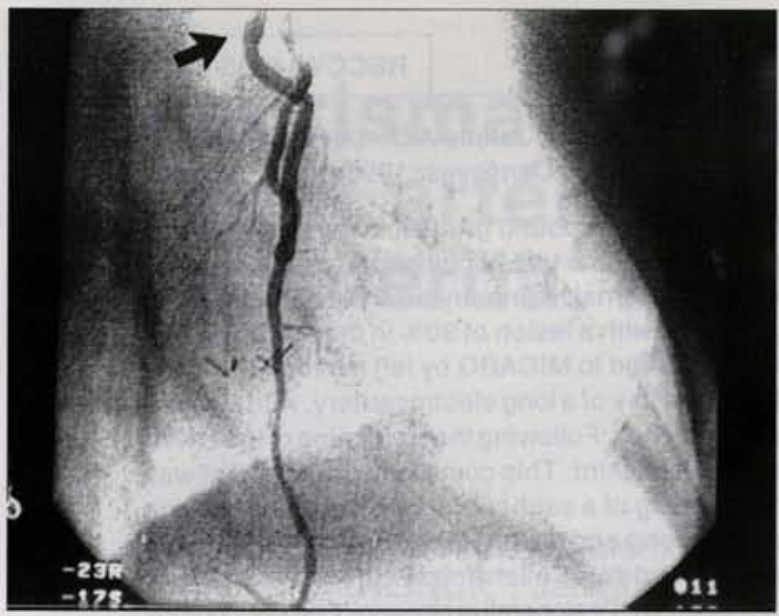

Fig. 2 - Angiografia pós-operatória revelando bom fluxo através do enxerto para a coronária. No detalhe (seta), a transição entre a artéria torácica interna e o segmento de veia safena.

angioplastia percutânea intracoronária tem como complicação não freqüente a oclusão coronária aguda, mas apresenta alto índice de reestenose, que ocorre em cerca de $30 \%$ dos pacientes nos primeiros 6 meses e $15 \%$ a partir do segundo ano, em especial em lesões proximais da artéria interventricular anterior $(7,8)$. Devido a este fato, os hemodinamicistas têm, mais recentemente, associado o uso de stents, os quais já estão começando a apresentar altos indices de recidivas ocorrendo em $6,9 \%$ dos pacientes com oclusão aguda entre 2 e 22 dias após o procedimento, $16 \%$ em procedimentos de emergência e $29,6 \%$ algum grau de reestenose (9), com seguimento tardio ainda não conhecido. Da mesma forma; relatam que $5 \%$ dos pacientes que são levados a angioplastia percutânea transluminal requerem cirurgia de revascularização de urgência por dissecção ou oclusão da coronária no momento do procedimento, ou dentro das 12 ou 24 horas. Os mecanismos de oclusão arterial incluem hemorragia subintimal, dissecção da camada intima da artéria junto à placa arterioesclerótica, espasmo coronário, trombose local, ou associação destes eventos. Dentro dos enxertos utilizados para revascularização do miocárdio, a artéria torácica interna é considerada o enxerto ideal para o tratamento cirúrgico da insuficiência coronária $(1,10)$ mantendo-se patente imediata e tardiamente, em comparação com outros enxertos, em especial a veia safena, principalmente quando utilizados para a artéria interventricular anterior. Esse fato tem determinado maior sobrevida tardia, menor incidência de eventos cardíacos, além de menor necessidade de reoperações.

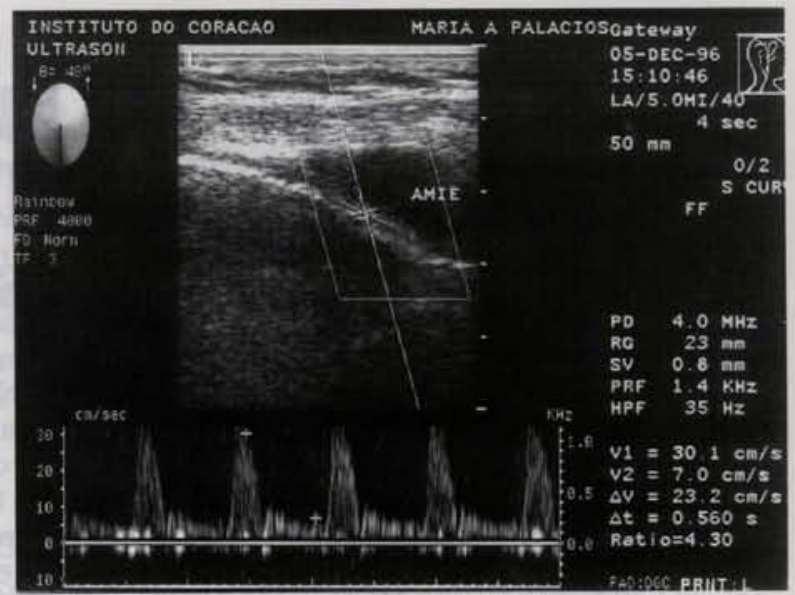

Fig. 3 - Ultrassonografia duplex de artéria mamária interna esquerda $-2^{2}$ espaço intercostal esquerdo, corte longitudinal. Acima observa-se segmento de artéria mamária esquerda com fluxo anterógrado. Abaixo observa-se curva da velocidade que indica leito distal de alta resistência (velocidade diastólica marcadamente reduzida: $V=7,0 \mathrm{~cm} / \mathrm{seg}$.)

Em recentes publicaçōes $(2,3)$, uma alternativa para revascularização do miocárdio é a cirurgia minimamente invasiva para o tratamento de artéria interventricular anterior, com bons resultados iniciais. BENETI (11) publicou, em 1995, uma alternativa para revascularização do miocárdio vídeoassistida com uso de toracoscópio, sem circulação extracorpórea. CALAFIORE (2), em 1996, descreveu tratamento de artéria interventricular anterior, utilizando minitoracotomia esquerda e também o uso de enxerto composto, com artéria epigástrica inferior para a extensão da artéria torácica interna esquerda $(12,14)$. Experiência similar foi relatada por LOBO FILHO et al. (4), entre nós, em recente publicação.

Em nossa experiência de 36 pacientes nos quais foi utilizada a CRMMI com anastomose da artéria torácica interna esquerda para a artéria interventricular anterior, o resultado imediato e a curto prazo tem sido bom. Neste paciente associamos um enxerto composto, devido à necessidade de alongamento da artéria torácica externa, com bom resultado imediato e a médio prazo.

Portanto, o uso de enxerto composto para revascularização do miocárdio na cirurgia minimamente invasiva é uma alternativa eficaz para ampliar a extensão da artéria torácica interna esquerda e possibilitar a anastomose, quando esta artéria é curta, ou apresenta problemas de fluxo, como na nossa paciente ou, ainda, se pretende tratar mais do que uma coronária. 
Jatene F B, Pêgo-Fernandes P, Arbulu H D, Hayata A LS, Kalil R, Molnar L, Jatene A D - Cirurgia de revascularização do miocárdio minimamente invasiva com uso de enxerto composto: relato de caso. Rev Bras Cir Cardiovasc 1996; 11 (4): $307-10$.

RBCCV $44205-325$

Jatene F B, Pêgo-Fernandes P, Arbulu H D, Hayata A L S, Kalil R, Molnar L, Jatene A D - Use of compound grafts in minimally invasive coronary artery bypass graft. Rev Bras Cir Cardiovasc 1996; 11 (4): $307-10$.

ABSTRACT: This paper reports our experience with the use of compound grafts in minimally invasive coronary artery bypass graft (MICABG). An alternative for MICABG is the use of compound grafts in cases where several coronary branches are to be revascularized, when internal mammary artery is demonstrated to be short, or branches off early. One female patient, 54 years old, with a lesion of $90 \%$ in the third proximal part of the left descending artery (LAD), was operated upon, submitted to MICABG by left minithoracotomy. The left internal mammary artery (LIMA) was amply dissected by the use of a long electrocautery, with the help of a videothoracoscope, and a clip for ligation of the intercostal branches. Following the sectioning of the LIMA, there was absence of blood flow and technical problems at the distal third. This compromised segment was disposed off and the LIMA was lengthened using the interpositioning of a saphenous vein segment, making possible the anastomosis with the LAD. Surgery was three hours long and the patient extubated fours hours postoperatively; the left pleural drainage tube was removed on the third day. Patient follow-up was satisfactory, without clinical problems; patient left the hospital in the fifth postoperative day. Postoperative cineangiocoronariography showed anastomosis patency; echodopplercardiogram performed on the fourth postoperative month showed patency and high resistence flow. In conclusion, the use of compound grafts for MICABG showed to be an efficient alternative to amplify the extension of the LIMA, enabling anastomosis when this artery is short or presents flow problems, or even if there is intention of treating more than one coronary artery.

DESCRIPTORS: Myocardial revascularization, methods. Thoracic arteries, surgery. Coronary vessels, surgery. Thoracoscopy. Surgery, minimally invasive.

\section{REFERÊNCIAS BIBLIOGRÁFICAS}

1 Loop F D, Lytle B W, Cosgrove D M et al. - Influence of the internal mammary artery on 10 years survival and other cardiac events. N Engl J Med 1986; 314:1-6.

2 Calafiore M A - Left anterior descending coronary artery grafting via left anterior small thoracotomy without cardiopulmonary bypass. Ann Thorac Surg 1996; 61: $1658-65$.

3 Acuff T E, Landreneau R J, Griffith B P, Mack M J Minimally invasive coronary artery bypass grafting. Ann Thorac Surg 1996; 61: 135-7.

4 Lobo Filho J G, Oliveira F M, Carline C et al. - Cirurgia de revascularizaçāo do miocárdio através de minitoracotomia ântero-lateral esquerda. Rev Bras Cir Cardiovasc 1996; 11: 143-7.

5 Buffolo E, Andrade J C S, Branco J N, Teles C A, Aguiar L F, Gomes W J - Coronary artery grafting without cardiopulmonary bypass. Ann Thorac Surg 1996; 61: 63-6.

6 Pfister A J , Zaki M S, Garcia J M et al. - Coronary artery bypass without cardiopulmonary bypass. Ann Thorac Surg 1992; 54: 1085-92.

7 Hueb W A - The Medicine Angioplasty or Surgery Study (MASS): a prospective randomized trial of medical therapy, balloon angioplasty or bypass surgery for single proximal left anterior descending artery stenoses. J Am Coll Cardiol 1995; 26: 1600-5.

8 Benetti F J - Coronary artery bypass surgery without extracorporeal circulation versus percutaneous transluminal coronary angioplasty: comparison of cost. J Thorac Cardiovasc Surg 1991; 102: 802-3.

9 Schoming A, Kastrati A, Mudra $\mathrm{H}$ et al. - Four-year experience with Palmaz-Schatz stenting in coronary angioplasty complicated by dissection with thretened or present vessel closure. Circulation 1994; 90: 2716-23.

10 Tector A J - Complete revascularization with internal thoracic artery grafts. Semin Thorac Cardiovasc Surg 1996; 8: 29-41.

11 Benetti F J - Video assisted coronary bypass surgery. J Cardiac Surg 1995; 10: 620-5.

12 Calafiore M A - Radial artery and inferior epigastric artery in composite grafts: improved midterm angiographic results. Ann Thorac Surg 1995; 60: 517-24.

13 Calafiore M A - Complete revascularization with three or more arterial conduits. Semin Thorac Cardiovasc Surg 1996; 8: 15-23.

14 Calafiore M A - Composite arterial conduits for a wider arterial myocardial revascularization. Ann Thorac Surg 1994; 58: 185-90. 